\title{
A curious differential calculus on the quantum disc and cones
}

\author{
Tomasz Brzeziński and Ludwik Dąbrowski
}

\begin{abstract}
A non-classical differential calculus on the quantum disc and cones is constructed and the associated integral is calculated.
\end{abstract}

Mathematics Subject Classification (2010). Primary 58B32.

Keywords. non-commutative geometry; differential forms; integral forms.

\section{Introduction}

The aim of this note is to present a two-dimensional differential calculus on the quantum disc algebra, which has no counterpart in the classical limit, but admits a well-defined (albeit different from the one in [2]) integral, and restricts properly to the quantum cone algebras. In this way the results of [3] are extended to other classes of non-commutative surfaces and to higher forms. The presented calculus is associated to an orthogonal pair of skewderivations, which arise as a particular example of skew-derivations on generalized Weyl algebras constructed recently in [1. It is also a fundamental ingredient in the construction of the Dirac operator on the quantum cone 6] that admits a twisted real structure in the sense of [5].

The reader unfamiliar with non-commutative differential geometry notions is referred to [4].

\section{A differential calculus on the quantum disc}

Let $0<q<1$. The coordinate algebra of the quantum disc, or the quantum disc algebra $\mathcal{O}\left(D_{q}\right)$ [8] is a complex *-algebra generated by $z$ subject to

$$
z^{*} z-q^{2} z z^{*}=1-q^{2} \text {. }
$$

To describe the algebraic contents of $\mathcal{O}\left(D_{q}\right)$ it is convenient to introduce a self-adjoint element $x=1-z z^{*}$, which $q^{2}$-commutes with the generator of $\mathcal{O}\left(D_{q}\right), x z=q^{2} z x$. A linear basis of $\mathcal{O}\left(D_{q}\right)$ is given by monomials $x^{k} z^{l}, x^{k} z^{* l}$. We view $\mathcal{O}\left(D_{q}\right)$ as a $\mathbb{Z}$-graded algebra, $\operatorname{setting} \operatorname{deg}(z)=1$, 
$\operatorname{deg}\left(z^{*}\right)=-1$. Associated with this grading is the degree-counting automorphism $\sigma: \mathcal{O}\left(D_{q}\right) \rightarrow \mathcal{O}\left(D_{q}\right)$, defined on homogeneous $a \in \mathcal{O}\left(D_{q}\right)$ by $\sigma(a)=q^{2 \operatorname{deg}(a)} a$. As explained in [1] there is an orthogonal pair of skewderivations $\partial, \bar{\partial}: \mathcal{O}\left(D_{q}\right) \rightarrow \mathcal{O}\left(D_{q}\right)$ twisted by $\sigma$ and given on the generators of $\mathcal{O}\left(D_{q}\right)$ by

$$
\partial(z)=z^{*}, \quad \partial\left(z^{*}\right)=0, \quad \bar{\partial}(z)=0, \quad \bar{\partial}\left(z^{*}\right)=q^{2} z,
$$

and extended to the whole of $\mathcal{O}\left(D_{q}\right)$ by the (right) $\sigma$-twisted Leibniz rule. Therefore, there is also a corresponding first-order differential calculus $\Omega^{1}\left(D_{q}\right)$ on $\mathcal{O}\left(D_{q}\right)$, defined as follows.

As a left $\mathcal{O}\left(D_{q}\right)$-module, $\Omega^{1}\left(D_{q}\right)$ is freely generated by one forms $\omega, \bar{\omega}$. The right $\mathcal{O}\left(D_{q}\right)$-module structure and the differential $d: \mathcal{O}\left(D_{q}\right) \rightarrow \Omega^{1}\left(D_{q}\right)$ are defined by

$$
\omega a=\sigma(a) \omega, \quad \bar{\omega} a=\sigma(a) \bar{\omega}, \quad d(a)=\partial(a) \omega+\bar{\partial}(a) \bar{\omega} .
$$

In particular,

$$
d z=z^{*} \omega=q^{2} \omega z^{*}, \quad d z^{*}=q^{2} z \bar{\omega}=\bar{\omega} z,
$$

and so, by the commutation rules (2.3),

$$
\omega=\frac{q^{-2}}{1-q^{2}}\left(d z z-q^{4} z d z\right), \quad \bar{\omega}=\frac{q^{-2}}{1-q^{2}}\left(z^{*} d z^{*}-q^{2} d z^{*} z^{*}\right) .
$$

Hence $\Omega^{1}\left(D_{q}\right)=\left\{\sum_{i} a_{i} d b_{i} \mid a_{i}, b_{i} \in \mathcal{O}\left(D_{q}\right)\right\}$, i.e. $\left(\Omega^{1}\left(D_{q}\right), d\right)$ is truly a firstorder differential calculus not just a degree-one part of a differential graded algebra. The appearance of $q^{2}-1$ in the denominators in (2.5) indicates that this calculus has no classical (i.e. $q=1$ ) counterpart.

The first-order calculus $\left(\Omega^{1}\left(D_{q}\right), d\right)$ is a $*$-calculus in the sense that the *-structure extends to the bimodule $\Omega^{1}\left(D_{q}\right)$ so that $(a \nu b)^{*}=b^{*} \nu^{*} a^{*}$ and $(d a)^{*}=d\left(a^{*}\right)$, for all $a, b \in \mathcal{O}\left(D_{q}\right)$ and $\nu \in \Omega^{1}\left(D_{q}\right)$, provided $\omega^{*}=\bar{\omega}$ (this choice of the $*$-structure justifies the appearance of $q^{2}$ in the definition of $\bar{\partial}$ in equation (2.2) $)$. From now on we view $\left(\Omega^{1}\left(D_{q}\right), d\right)$ as a $*$-calculus, which allows us to reduce by half the number of necessary checks.

Next we aim to show that the module of 2 -forms $\Omega^{2}\left(D_{q}\right)$ obtained by the universal extension of $\Omega^{1}\left(D_{q}\right)$ is generated by the anti-self-adjoint 2-form 1

$$
\mathrm{v}=\frac{q^{-6}}{q^{2}-1}\left(\omega^{*} \omega+q^{8} \omega \omega^{*}\right), \quad \mathrm{v}^{*}=-\mathrm{v}
$$

and to describe the structure of $\Omega^{2}\left(D_{q}\right)$. By (2.3), for all $a \in \mathcal{O}\left(D_{q}\right)$,

$$
\mathrm{v} a=\sigma^{2}(a) \mathrm{v} \text {. }
$$

Combining commutation rules (2.3) with the relations (2.4) we obtain

$$
z^{*} d z=q^{2} d z z^{*}, \quad d z z-q^{4} z d z=q^{2}\left(1-q^{2}\right) \omega,
$$

\footnotetext{
${ }^{1}$ One should remember that the $*$-conjugation takes into account the parity of the forms; see 9 .
} 
and their $*$-conjugates. The differentiation of the first of equations (2.8) together with (2.3) and (2.1) yield

$$
\omega \omega^{*}=(1-x) \mathrm{v}, \quad \omega^{*} \omega=q^{6}\left(q^{2} x-1\right) \mathrm{v},
$$

which means that $\omega \omega^{*}$ and $\omega^{*} \omega$ are in the module generated by $v$. Next, by differentiating $\omega z^{*}=q^{-2} z^{*} \omega$ and $\omega z=q^{2} z \omega$ and using (2.4) and (2.3) one obtains

$$
d \omega z^{*}=q^{-2} z^{*} d \omega+z\left(\omega^{*} \omega+q^{4} \omega \omega^{*}\right), \quad d \omega z=q^{2} z d \omega+\left(q^{2}+q^{-2}\right) z^{*} \omega^{2} .
$$

The differentiation of $d z=z^{*} \omega$ yields

$$
z^{*} d \omega=-q^{2} z \omega^{*} \omega
$$

Multiplying this relation by $z$ from left and right, and using commutation rules (2.1) and (2.3) one finds that $(1-x) d \omega=q^{-4} z^{*} d \omega z$. Developing the right hand side of this equality with the help of the second of equations (2.10) we find

$$
d \omega=\frac{1+q^{-4}}{q^{2}-1} z^{* 2} \omega^{2} .
$$

Combining (2.10) with (2.12) we can derive

$$
z^{* 3} \omega^{2}=-z \frac{q^{8}}{q^{4}+1}\left(\omega^{*} \omega+q^{4} \omega \omega^{*}\right) .
$$

The multiplication of (2.13) by $z^{3}$ from the left and right and the usage of (2.1), (2.3) give

$$
\begin{aligned}
& (1-x)\left(1-q^{-2} x\right)\left(1-q^{-4} x\right) \omega^{2}=-\frac{q^{8}}{q^{4}+1} z^{4}\left(\omega^{*} \omega+q^{4} \omega \omega^{*}\right), \\
& \left(1-q^{2} x\right)\left(1-q^{4} x\right)\left(1-q^{6} x\right) \omega^{2}=-\frac{q^{8}}{q^{4}+1} z^{4}\left(\omega^{*} \omega+q^{4} \omega \omega^{*}\right) .
\end{aligned}
$$

Comparing the left hand sides of equations (2.14), we conclude that

$$
x \omega^{2}=0=\omega^{2} x \quad \text { and, by } * \text {-conjugation, } \quad x \omega^{* 2}=0=\omega^{* 2} x,
$$

and hence in view of either of (2.14)

$$
\omega^{2}=-\frac{q^{8}}{q^{4}+1} z^{4}\left(\omega^{*} \omega+q^{4} \omega \omega^{*}\right) .
$$

By (2.9), the right hand side of (2.16) is in the module generated by $v$, and so is $\omega^{2}$ and its adjoint $\omega^{* 2}$. Thus, the module $\Omega^{2}\left(D_{q}\right)$ spanned by all products of pairs of one-forms is indeed generated by $\mathrm{v}$.

Multiplying (2.12) and (2.11) by $x$ and using relations (2.15) we obtain

$$
x z \omega^{*} \omega=0=\omega^{*} \omega x z .
$$

Following the same steps but now starting with the differentiation of $d z^{*}=$ $q^{2} z \omega^{*}$ (see (2.4)), we obtain the complementary relation

$$
x z \omega \omega^{*}=0=\omega \omega^{*} x z .
$$

In view of the definition of $\mathrm{v},(2.17)$ and (2.18) yield $x z \mathrm{v}=0=\mathrm{v} x z$. Next, the multiplication of, say, the first of these equations from the left and right 
by $z^{*}$ and the use of (2.1) yield $x(1-x) \mathrm{v}=0$ and $x\left(1-q^{2} x\right) \mathrm{v}=0$. The subtraction of one of these equations from the suitable scalar multiple of the other produces the necessary relation

$$
x \mathrm{v}=0=\mathrm{v} x,
$$

which fully characterises the structure of $\Omega^{2}\left(D_{q}\right)$ as an $\mathcal{O}\left(D_{q}\right)$-module generated by v. In the light of (2.19), the $\mathbb{C}$-basis of $\Omega^{2}\left(D_{q}\right)$ consists of elements $\mathrm{v} z^{n}, \mathrm{v} z^{* m}$, and hence, for all $w \in \Omega^{2}\left(D_{q}\right), w x=x w=0$, i.e., $\Omega^{2}\left(D_{q}\right)$ is a torsion (as a left and right $\mathcal{O}\left(D_{q}\right)$-module). Since $\mathcal{O}\left(D_{q}\right)$ is a domain and $\Omega^{2}\left(D_{q}\right)$ is a torsion, the dual of $\Omega^{2}\left(D_{q}\right)$ is the zero module, hence, in particular $\Omega^{2}\left(D_{q}\right)$ is not projective. Again by (2.19), the annihilator of $\Omega^{2}\left(D_{q}\right)$,

$$
\operatorname{Ann}\left(\Omega^{2}\left(D_{q}\right)\right):=\left\{a \in \mathcal{O}\left(D_{q}\right) \mid \forall w \in \Omega^{2}\left(D_{q}\right), a w=w a=0\right\},
$$

is the ideal of $\mathcal{O}\left(D_{q}\right)$ generated by $x$. The quotient $\mathcal{O}\left(D_{q}\right) / \operatorname{Ann}\left(\Omega^{2}\left(D_{q}\right)\right)$ is the Laurent polynomial ring in one variable, i.e. the algebra $\mathcal{O}\left(S^{1}\right)$ of coordinate functions on the circle. When viewed as a module over $\mathcal{O}\left(S^{1}\right), \Omega^{2}\left(D_{q}\right)$ is free of rank one, generated by v. Thus, although the module of 2 -forms over $\mathcal{O}\left(D_{q}\right)$ is neither free nor projective, it can be identified with sections of a trivial line bundle once pulled back to the (classical) boundary of the quantum disc.

With (2.19) at hand, equations (2.9), (2.16), (2.12) and their $*$-conjugates give the following relations in $\Omega^{2}\left(D_{q}\right)$

$$
\begin{array}{r}
d \omega=q^{8} z^{2} \mathrm{v}, \quad d \omega^{*}=-z^{* 2} \mathrm{v}, \quad \omega \omega^{*}=\mathrm{v}, \quad \omega^{*} \omega=-q^{6} \mathrm{v}, \\
\omega^{2}=q^{12} \frac{q^{2}-1}{q^{4}+1} z^{4} \mathrm{v}, \quad \omega^{* 2}=q^{-4} \frac{q^{2}-1}{q^{4}+1} z^{* 4} \mathrm{v} .
\end{array}
$$

One can easily check that (2.20), (2.19) and (2.7) are consistent with (2.3) with no further restrictions on $\mathrm{v}$. Setting $\Omega^{n}\left(D_{q}\right)=0$, for all $n>2$, we thus obtain a 2-dimensional calculus on the quantum disc.

\section{Differential calculus on the quantum cone}

The quantum cone algebra $\mathcal{O}\left(C_{q}^{N}\right)$ is a subalgebra of $\mathcal{O}\left(D_{q}\right)$ consisting of all elements of the $\mathbb{Z}$-degree congruent to 0 modulo a positive natural number $N$. Obviously $\mathcal{O}\left(C_{q}^{1}\right)=\mathcal{O}\left(D_{q}\right)$, the case we dealt with in the preceding section, so we may assume $N>1$. $\mathcal{O}\left(C_{q}^{N}\right)$ is a $*$-algebra generated by the self-adjoint $x=1-z z^{*}$ and by $y=z^{N}$, which satisfy the following commutation rules

$$
x y=q^{2 N} y x, \quad y y^{*}=\prod_{l=0}^{N-1}\left(1-q^{-2 l} x\right), \quad y^{*} y=\prod_{l=1}^{N}\left(1-q^{2 l} x\right) .
$$

The calculus $\Omega\left(C_{q}^{N}\right)$ on $\mathcal{O}\left(C_{q}^{N}\right)$ is obtained by restricting of the calculus $\Omega\left(D_{q}\right)$, i.e. $\Omega^{n}\left(C_{q}^{N}\right)=\left\{\sum_{i} a_{0}^{i} d\left(a_{1}^{i}\right) \cdots d\left(a_{n}^{i}\right) a_{n+1}^{i} \mid a_{k}^{i} \in \mathcal{O}\left(C_{q}^{N}\right)\right\}$. Since $d$ is a degree-zero map $\Omega\left(C_{q}^{N}\right)$ contains only these forms in $\Omega\left(D_{q}\right)$, whose $\mathbb{Z}$-degree is a multiple of $N$. We will show that all such forms are in $\Omega\left(C_{q}^{N}\right)$. Since $\operatorname{deg}(\omega)=2, \operatorname{deg}\left(\omega^{*}\right)=-2$ and $\operatorname{deg}(\mathrm{v})=0$, this is equivalent to

$$
\Omega^{1}\left(C_{q}^{N}\right)=\mathcal{O}\left(D_{q}\right) \overline{-2} \omega \oplus \mathcal{O}\left(D_{q}\right)_{\overline{2}} \omega^{*}, \quad \Omega^{2}\left(C_{q}^{N}\right)=\mathcal{O}\left(C_{q}^{N}\right) \vee
$$


where $\mathcal{O}\left(D_{q}\right)_{\bar{s}}=\left\{a \in \mathcal{O}\left(D_{q}\right) \mid \operatorname{deg}(a) \equiv s \bmod N\right\}$.

As an $\mathcal{O}\left(C_{q}^{N}\right)$-module, $\mathcal{O}\left(D_{q}\right) \frac{}{-2}$ is generated by $z^{N-2}$ and $z^{* 2}$, hence to show that $\mathcal{O}\left(D_{q}\right)_{-2} \omega \subseteq \Omega^{1}\left(C_{q}^{N}\right)$ suffices it to prove that $z^{N-2} \omega, z^{* 2} \omega \in$ $\Omega^{1}\left(C_{q}^{N}\right)$. Using the Leibniz rule one easily finds that

$$
d y=\left(\left[N ; q^{2}\right]-q^{-2 N+4}\left[N ; q^{4}\right] x\right) z^{N-2} \omega,
$$

where $[n ; s]:=\frac{s^{n}-1}{s-1}$. Hence, in view of (2.1) and (2.3),

$$
\begin{gathered}
y^{*} d y=\left[N ; q^{2}\right]\left(1-q^{4} \frac{\left[N ; q^{4}\right]}{\left[N ; q^{2}\right]} x\right) \prod_{l=3}^{N}\left(1-q^{2 l} x\right) z^{* 2} \omega \\
d y y^{*}=q^{-2 N}\left[N ; q^{2}\right]\left(1-q^{-2 N+4} \frac{\left[N ; q^{4}\right]}{\left[N ; q^{2}\right]} x\right) \prod_{l=0}^{N-3}\left(1-q^{-2 l} x\right) z^{* 2} \omega .
\end{gathered}
$$

The polynomial in $x$ on the right hand side of (3.2a) has roots in common with the polynomial on the right hand side of (3.2b) if and only if there exists an integer $k \in[-2 N+2,-N-1] \cup[2, N-1]$ such that

$$
q^{2 k}\left(q^{2 N}+1\right)=q^{2}+1
$$

Equation (3.3) is equivalent to $q^{2}\left[N+k-1 ; q^{2}\right]+\left[k ; q^{2}\right]=0$, with the left hand side strictly positive if $k>0$ and strictly negative if $k \leq-N$. So, there are no solutions within the required range of values of $k$. Hence the polynomials (3.2a), (3.2b) are coprime, and so there exists a polynomial (in $x)$ combination of the left had sides of equations (3.2) that gives $z^{* 2} \omega$. This combination is an element of $\Omega^{1}\left(C_{q}^{N}\right)$ and so is $z^{* 2} \omega$. Next,

$$
\begin{gathered}
z^{* 2} \omega y=q^{2 N}\left(1-q^{2} x\right)\left(1-q^{4} x\right) z^{N-2} \omega, \\
y z^{* 2} \omega=\left(1-q^{-2 N+4} x\right)\left(1-q^{-2 N+2} x\right) z^{N-2} \omega,
\end{gathered}
$$

so again there is an $x$-polynomial combination of the left hand sides (which are already in $\left.\Omega^{1}\left(C_{q}^{N}\right)\right)$ giving $z^{N-2} \omega$. Therefore, $\mathcal{O}\left(D_{q}\right)_{-2} \omega \subseteq \Omega^{1}\left(C_{q}^{N}\right)$. The case of $\mathcal{O}\left(D_{q}\right)_{2}$ follows by the $*$-conjugation.

Since $z^{2} \omega^{*}, z^{* 2} \omega$ are elements of $\Omega^{1}\left(C_{q}^{N}\right)$,

$$
\Omega^{2}\left(C_{q}^{N}\right) \ni z^{2} \omega^{*} z^{* 2} \omega=q^{-4}(1-x)\left(1-q^{-2} x\right) \omega^{*} \omega=-q^{2} \mathrm{v},
$$

by the quantum disc relations and (2.20) and 2.19). Consequently, $v \in$ $\Omega^{2}\left(C_{q}^{N}\right)$. Therefore, $\Omega\left(C_{q}^{N}\right)$ can be identified with the subspace of $\Omega\left(D_{q}\right)$, of all the elements whose $\mathbb{Z}$-degree is a multiple of $N$.

\section{The integral}

Here we construct an algebraic integral associated to the calculus constructed in Section 2. We start by observing that since $\sigma$ preserves the $\mathbb{Z}$-degrees of elements of $\mathcal{O}\left(D_{q}\right)$ and $\partial$ and $\bar{\partial}$ satisfy the $\sigma$-twisted Leibniz rules, the definition (2.2) implies that $\partial$ lowers while $\bar{\partial}$ raises degrees by 2 . Hence, one can equip $\Omega^{1}\left(D_{q}\right)$ with the $\mathbb{Z}$-grading so that $d$ is the degree zero map, 
provided $\operatorname{deg}(\omega)=2, \operatorname{deg}\left(\omega^{*}\right)=-2$. Furthermore, in view of the definition of $\sigma$, one easily finds that

$$
\sigma^{-1} \circ \partial \circ \sigma=q^{4} \partial, \quad \sigma^{-1} \circ \bar{\partial} \circ \sigma=q^{-4} \bar{\partial},
$$

i.e. $\partial$ is a $q^{4}$-derivation and $\bar{\partial}$ is a $q^{-4}$-derivation. Therefore, by [7], $\Omega\left(D_{q}\right)$ admits a divergence, for all right $\mathcal{O}\left(D_{q}\right)$-linear maps $f: \Omega^{1}\left(D_{q}\right) \rightarrow \mathcal{O}\left(D_{q}\right)$, given by

$$
\nabla_{0}(f)=q^{4} \partial(f(\omega))+q^{-4} \bar{\partial}\left(f\left(\omega^{*}\right)\right) .
$$

Since the $\mathcal{O}\left(D_{q}\right)$-module $\Omega^{2}\left(D_{q}\right)$ has a trivial dual, $\nabla_{0}$ is flat. Recall that by the integral associated to $\nabla_{0}$ we understand the cokernel map of $\nabla_{0}$.

Theorem 4.1. The integral associated to the divergence (4.2) is a map $\Lambda$ : $\mathcal{O}\left(D_{q}\right) \rightarrow \mathbb{C}$, given by

$$
\Lambda\left(x^{k} z^{l}\right)=\lambda \frac{\left[k+1 ; q^{2}\right]}{\left[k+1 ; q^{4}\right]} \delta_{l, 0}, \quad \text { for all } k \in \mathbb{N}, l \in \mathbb{Z},
$$

where, for $l<0$, $z^{l}$ means $z^{*-l}$ and $\lambda \in \mathbb{C}$.

Proof. First we need to calculate the image of $\nabla_{0}$. Using the twisted Leibniz rule and the quantum disc algebra commutation rules (2.1), one obtains

$$
\partial\left(x^{k}\right)=-q^{-2}\left[k ; q^{4}\right] x^{k-1} z^{* 2} .
$$

Since $\partial\left(z^{*}\right)=0$, (4.4) means that all monomials $x^{k} z^{* l+2}$ are in the image of $\partial$ hence in the image of $\nabla_{0}$. Using the $*$-conjugation we conclude the $x^{k} z^{l+2}$ are in the image of $\bar{\partial}$ hence in the image of $\nabla_{0}$. So $\Lambda$ vanishes on (linear combinations of) all such polynomials. Next note that

$$
\partial\left(z^{2}\right)=\left(q^{2}+1\right)-\left(q^{4}+1\right) x,
$$

hence

$$
\partial\left(z^{*} z^{2}-q^{4} z^{2} z^{*}\right)=\left(1-q^{4}\right) z^{*}, \quad \partial\left(z^{*} z^{2}-q^{2} z^{2} z^{*}\right)=\left(1-q^{2}\right)\left(1+q^{4}\right) x z^{*} .
$$

This means that $z^{*}$ and $x z^{*}$ are in the image of $\partial$, hence of $\nabla_{0}$. In fact, all the $x^{k} z^{*}$ are in this image which can be shown inductively. Assume $x^{k} z^{*} \in \operatorname{Im}(\partial)$, for all $k \leq n$. Then using the twisted Leibniz rule, (4.4) and (4.5) one finds

$$
\partial\left(x^{n} z^{2}\right)=-q^{2}\left[N ; q^{4}\right] x^{n-1}+\left(q^{2}+1\right)\left[n+1 ; q^{4}\right] x^{n}-\left[n+2 ; q^{4}\right] x^{n+1} \text {. }
$$

Since $\partial\left(z^{*}\right)=0$, equation (4.6) implies that $\partial\left(z^{n} z^{2} z^{*}\right)$ is a linear combination of monomials $x^{n-1} z^{*}, x^{n} z^{*}$ and $x^{n+1} z^{*}$. Since the first two are in the image of $\partial$ by the inductive assumption, so is the third one. Therefore, all linear combinations of $x^{k} z^{*}$ and $x^{k} z$ (by the $*$-conjugation) are in the image of $\nabla_{0}$.

Put together all this means that $\Lambda$ vanishes on all the polynomials $\sum_{k, l=1}^{n}\left(c_{k l} x^{k} z^{l}+c_{k l}^{\prime} x^{k} z^{* l}\right)$. The rest of the formula (4.3) can be proven by induction. Set $\lambda=\Lambda(1)$. Since $\Lambda$ vanishes on all elements in the image of $\nabla_{0}$, hence also in the image of $\partial$, the application of $\Lambda$ to the right hand side of (4.4) confirms (4.3) for $k=1$. Now assume that (4.3) is true for all $k \leq n$. 
Then the application $\Lambda$ to the right hand side of (4.6) followed by the use of the inductive assumption yields

$$
\begin{aligned}
{\left[n+2 ; q^{4}\right] \Lambda\left(x^{n+1}\right) } & =q^{2}\left[N ; q^{4}\right] \Lambda\left(x^{n-1}\right)-\left(q^{2}+1\right)\left[n+1 ; q^{4}\right] \Lambda\left(x^{n}\right) \\
& =\lambda\left(\left(q^{2}+1\right)\left[n+1 ; q^{2}\right]-q^{2}\left[n ; q^{2}\right]\right)=\lambda\left[n+2 ; q^{2}\right] .
\end{aligned}
$$

Therefore, the formula (4.3) is true also for $n+1$, as required.

The restriction of $\Lambda$ to the elements of $\mathcal{O}\left(D_{q}\right)$, whose $\mathbb{Z}$-degree is a multiple of $N$ gives an integral on the quantum cone $\mathcal{O}\left(C_{q}^{N}\right)$.

\section{Acknowledgment}

The work on this project began during the first author's visit to SISSA, supported by INdAM-GNFM. He would like to thank the members of SISSA for hospitality. The second author was supported in part by the Simons Foundation grant 346300 and the Polish Government MNiSW 2015-2019 matching fund.

\section{References}

[1] A. Almulhem \& T. Brzeziński, Skew derivations on generalized Weyl algebras. arXiv:1610.03282 (2016).

[2] E.J. Beggs \& S. Majid, Spectral triples from bimodule connections and Chern connections. J. Noncommut. Geom. to appear, arXiv:1508.04808v2, (2015).

[3] T. Brzeziński, Non-commutative differential geometry of generalized Weyl algebras. SIGMA Symmetry Integrability Geom. Methods Appl. 12 (2016), 059.

[4] T. Brzeziński, Differential and integral forms on non-commutative algebras. arXiv:1611.01016, (2016).

[5] T. Brzeziński, N. Ciccoli, L. Dąbrowski \& A. Sitarz, Twisted reality condition for Dirac operators. Math. Phys. Anal. Geom. 19 (2016), 19:16.

[6] T. Brzeziński \& L. Dąbrowski, In preparation.

[7] T. Brzeziński, L. El Kaoutit \& C. Lomp, Non-commutative integral forms and twisted multi-derivations. J. Noncommut. Geom. 4 (2010), 281-312.

[8] S. Klimek \& A. Lesniewski, A two-parameter quantum deformation of the unit disc. J. Funct. Anal. 115 (1993), 1-23.

[9] S.L. Woronowicz, Differential calculus on compact matrix pseudogroups (quantum groups). Comm. Math. Phys. 122 (1989), 125-170.

\section{Tomasz Brzeziński}

Department of Mathematics, Swansea University, Swansea SA2 8PP, U.K.

Department of Mathematics, University of Białystok, K. Ciołkowskiego 1M, 15-245

Białystok, Poland. E-mail: T.Brzezinski@swansea.ac.uk

Ludwik Dąbrowski

SISSA, Via Bonomea 265, 34136 Trieste, Italy. E-mail: dabrow@sissa.it 